\title{
Reintroducción de la circuncisión como una técnica quirúrgica reconstructiva
}

\section{Reintroduction of Circumcision as a Technique Reconstructive Surgical}

\author{
Camila Moreno Bencardino ${ }^{1(0)}$ Jose Nicolas Fernandez ${ }^{2}$ (1) \\ ${ }^{1}$ Residente de Urología, Pontificia Universidad Javeriana, Bogotá, Colombia \\ ${ }^{2}$ Urólogo Pediatra, Pontificia Universidad Javeriana, Bogotá, Colombia \\ Address for correspondence Maria Camila Moreno Bencardino, MD, \\ Residente de Urología, Pontificia Universidad Javeriana, Bogotá, \\ Colombia (e-mail: m.morenob93@gmail.com).
}

Urol Colomb 2021;30:77-79.

\section{Resumen \\ Palabras clave \\ - circuncisión \\ - reconstrucción peneana \\ - urología reconstructiva \\ - cicatrización}

La circuncisión es una cirugía practicada frecuentemente en urología. Sin tener en cuenta los principios básicos de la cirugía reconstructiva, puede generar procesos de cicatrización anómalos, con resultados estéticos y funcionales inadecuados, fimosis secundaria, cicatrización hipertrófica, queloide o "pene enterrado." El objetivo de este video es recordar la importancia de operar con las directrices de una cirugía reconstructiva, resaltando el cuidado de los tejidos y el uso de suturas de bajo calibre, para prevenir complicaciones y resultados insatisfactorios, resaltando la preservación de las zonas postuladas por Firlit. Se sospecha que las complicaciones de este procedimiento son subestimadas por los especialistas. El manejo cuidadoso de los tejidos, el uso de suturas de bajo calibre y los puntos subcuticulares con poca tensión, son medios para optimizar el proceso de cicatrización, evitando la isquemia y las cicatrices hipertróficas, basados en el fundamento de que esta es una cirugía no solo funcional, sino reconstructiva y estética. La circuncisión es un procedimiento que debe conservar la funcionalidad y estética del pene, por lo que debe ser realizado bajo las directrices de una cirugía reconstructiva.

Circumcision is a frequently practiced surgical procedure in urology. Without considering the basic principles of reconstructive surgery, it can result in abnormal cicatrization processes, with inadequate aesthetic or functional results, secondary phimosis, hypertrophic or keloid scarring, or even "buried penis." The objective of this video is to remind the importance of operating with the principles of reconstructive surgery, highlighting the careful handling of tissues and the use of low-caliber sutures, to prevent complications and unsatisfactory results, preserving the areas postulated by Firlit. The complications of this procedure are suspected to be underestimated by specialists. Careful tissue management, the use of low-caliber sutures and subcuticular points with no tension are means to optimize the healing process, avoiding ischemia and hypertrophic scars, based on the fact that this is a surgery that is not only functional, but reconstructive and aesthetic as well. Circumcision is a procedure that must preserve the functionality and aesthetics of the penis and it must be performed under the principles of reconstructive surgery. received

January 14, 2020

accepted

April 3, 2020

published online

July 20,2020
DOI https://doi.org/

$10.1055 / \mathrm{s}-0040-1712111$

ISSN $0120-789 X$

e ISSN 2027-0119.
(C) 2020. Sociedad Colombiana de Urología. All rights reserved.

This is an open access article published by Thieme under the terms of the

Creative Commons Attribution-NonDerivative-NonCommercial-License,

permitting copying and reproduction so long as the original work is given appropriate credit. Contents may not be used for commercial purposes, or adapted, remixed, transformed or built upon. (https://creativecommons.org/ licenses/by-nc-nd/4.0/)

Thieme Revinter Publicações Ltda., Rua do Matoso 170, Rio de Janeiro, RJ, CEP 20270-135, Brazil 


\section{Introducción}

La circuncisión es una cirugía practicada frecuentemente en urología. Sin tener en cuenta los principios básicos de la cirugía reconstructiva, puede generar procesos de cicatrización anómalos, con resultados estéticos y funcionales inadecuados, fimosis secundaria, cicatrización hipertrófica, queloide o "pene enterrado."1-4

El objetivo de este video, es recordar la importancia de operar con las directrices de una cirugía reconstructiva, resaltando el cuidado de los tejidos y el uso de suturas de bajo calibre, para prevenir complicaciones y resultados insatisfactorios.

\section{Descripción del Procedimiento}

Luego de la liberación cuidadosa de adherencias balanoprepuciales, se coloca un punto de reparo de PDS o prolene 4-0 en el glande, que permitirá la exposición, tracción y movilización durante el procedimiento. Recomendamos no usar agujas cortantes y pasar el punto de ventral a dorsal para reducir el sangrado glandular. La colocación del bloqueo del nervio dorsal del pene, con ayuda de la tracción del glande, se hace en la línea media evitando la movilización de la aguja para disminuir el riesgo de sangrado.

La marcación y planeación de los sitios de incisión es un paso importante ya que previene las irregularidades en el corte, y permite planear los colgajos de piel para reducir el pene oculto y el defecto en vela. Sugerimos preservar un segmento mayor de mucosa prepucial coronal, permitiendo conservar su fisiología, siguiendo los principios de las tres zonas postulados por Firlit. ${ }^{5}$ Se realiza una técnica de doble incisión y se reseca la mucosa prepucial redundante incluyendo el anillo fimótico con electrobisturí, lo que asegura una mejor hemostasia.

Para afrontar la piel, se usan puntos subcuticulares, con la suficiente tensión para afrontar los tejidos, pero no excesiva, para evitar isquemia y cicatrización hipertrófica. Sugerimos evitar puntos perforantes porque dejan cicatrices. Se colocan cuatro puntos cardinales de afrontamiento.

Finalmente se retira el punto de reparo glandular y se coloca un vendaje levemente compresivo.

\section{Discusión}

Este video busca resaltar la importancia de una técnica cuidadosa de circuncisión. Las complicaciones de este procedimiento se han descrito en la literatura, pero impresiona que son subestimadas por los especialistas. ${ }^{1}$ En una serie de Budapest, se reportan 48 hombres llevados a circuncisión con resultados insatisfactorios. El 44\% por cicatriz hipertrófica, el $13 \%$ por una línea de cicatrización irregular ("wrinkling"), el 11\% por piel redundante y el 3,6\% por parafimosis. ${ }^{6}$

El manejo cuidadoso de los tejidos, el uso de suturas de bajo calibre y los puntos subcuticulares con poca tensión, son medios para optimizar el proceso de cicatrización, basados en el fundamento de que esta es una cirugía no solo funcional, sino reconstructiva y estética. Estas son medidas que previenen la isquemia de los tejidos y la formación de cicatrices hipertróficas. El punto de reparo glandular es relevante porque permite la tracción, movilización y exposición de los tejidos, facilitando el procedimiento para el cirujano, sin repercusiones en el paciente. ${ }^{1,6-8}$

\section{Conclusión}

La circuncisión es un procedimiento que debe conservar la funcionalidad y estética del pene, por lo que debe ser realizado bajo las directrices de una cirugía reconstructiva.

\section{Video 1}

Online content including video sequences viewable at: https://www.thiemeconnect.com/products/ejournals/html/ doi/10-1055-s-0040-1712111-ruc-20-00004-v1.mp4.

Responsabilidades Éticas

El video cumple con los principios establecidos en la declaración de Helsinki de la AMM (Asociación Médica Mundial), inicialmente propuestos en 1964 y enmendados por última vez en octubre de 2013. Los autores declaran que no hay conflictos de interés en la publicación del video. Se han seguido las normas de confidencialidad y privacidad de los pacientes.

Se trata de un estudio de investigación sin riesgo, según la resolución 8430 de 1993, ya que hace parte de los estudios que emplean técnicas y métodos de investigación documental retrospectivos y aquellos en los que no se realiza ninguna intervención o modificación intencionada de las variables biológicas, fisiológicas, sicológicas o sociales de los individuos que participan en el estudio. Además, se respetará en todo momento la confidencialidad de los individuos y no se usarán datos que puedan identificarlos, en cumplimiento de la Ley 1581 de 2012. A lo largo de este estudio, no se vulnerarán los principios de autonomía, beneficencia, no maleficencia o justicia del paciente o su pareja. Por lo anterior, no se requiere un consentimiento informado.

Conflicto de Intereses

Los autores declaran no tener ningún conflicto de intereses.

\section{Referencias}

1 Williams N, Kapila L. Complications of circumcision. Br J Surg 1993;80(10):1231-1236

2 Fuller TW, Theisen KM, Shah A, Rusilko PJ. Surgical Management of Adult Acquired Buried Penis. Curr Urol Rep 2018;19(03):22

3 Pestana IA, Greenfield JM, Walsh M, Donatucci CF, Erdmann D. Management of "buried" penis in adulthood: an overview. Plast Reconstr Surg 2009;124(04):1186-1195 
4 Talini C, Antunes LA, Carvalho BCN, et al. Circumcision: postoperative complications that required reoperation. Einstein (Sao Paulo) 2018;16(03):eA04241

5 Kolligian ME, Firlit CF. The mucosal collar revisited. Urology 2000; 55(01):114-117

6 Fekete F, Török A, Nyirády P. Revisions after unsatisfactory adult circumcisions. Int Urol Nephrol 2011;43(02):431-435
7 Demirdover C, Sahin B, Vayvada H, Oztan HY. Keloid formation after circumcision and its treatment. J Pediatr Urol 2013;9(01): e54-e56. Doi: 10.1016/j.jpurol.2012.07.018 [Internet]

8 Smith JA, Howards SS, Preminger GM, Hinman F. Hinman's Atlas of urologic surgery. In: Wan J, editor. 3rd ed. Elsevier/Saunders; 2012:139-144 